\title{
Dentsply Sirona introduces a complete Class II solution
}

When placing a composite restoration, especially in Class II procedures, one of the key challenges is to achieve excellent adaptation. For optimum results, multiple products must adapt well to the prepared tooth, to the physical and chemical properties of the other products being used and to the technique of the dental practitioner.

Dentsply Sirona is addressing this challenge with three innovative products designed to work together while providing superior adaptation at every step of the restoration:

- Palodent Sectional Matrix System adapts to the natural contour of the tooth

- SDR Smart Dentine Replacement adapts to the shape of the cavity floor and matrix band, and self-levels to adapt to the final occlusal layer

- ceram.x universal the most complete nano-ceramic composite adapts to the cavity walls, SDR material and the practitioner's sculpting techniques.

Each of these products, alone, offers best-in-class convenience, handling and performance. Used together, they complement one another to provide a complete Class II solution with superior adaptability.

\section{Adapting to the requirements of each Class II step}

Class II restorations are among the most common treatments in dentistry, yet among the most challenging for achieving a natural shape, lifelike aesthetics and long-term durability. In research with a wide range of dental practitioners and key opinion leaders, Dentsply Sirona has identified three essential features of a successful restoration that are directly affected by the products used and their adaptability to the dentist's technique and the requirements of each Class II case.

- The restoration must re-establish the tooth's natural contour and interproximal contact. If the interproximal contact is improperly shaped, poorly placed or left open, the restoration can fail due to fracture, food impaction, periodontal inflammation or recurrent caries. The Palodent V3 system is easy to use and adapts to the natural shape of the tooth to provide predictable, anatomically correct contacts and tight gingival seals with less flash and finishing

- The fill material must adapt to the prepared surface. If the restorative material does not fully conform to the shape of the cavity preparation, any unfilled areas and voids can result in postoperative sensitivity, discolouration and recurrent caries. SDR material can be used to bulk-fill up to $4 \mathrm{~mm}$, flowing readily and self-levelling to adapt fully to the proximal margins and matrix band while saving up to $40 \%$ in placement time. With up to $60 \%$ less shrinkage stress, SDR material has been clinically proven in a five-year study to provide the same durability and aesthetic as fill materials that must be applied in multiple layers ${ }^{1}$

- Lifelike aesthetics and proper occlusal anatomy depend on the sculptability, stickiness and shade matching. ${ }^{2}$ Unsatisfactory material properties can lead to additional handling effort, prolonged placement and sculpting, additional finishing and polishing, and poor aesthetic outcomes. ceram.x universal restorative adapts efficiently to the cavity walls and the SDR layer beneath it. Its innovative Sphere TEC Technology combines adaptive consistency with excellent slump-resistance and easy sculptability. ceram.x universal exhibits a distinct chameleon effect in a simple shading system that covers the full VITA ${ }^{3}$ range with only five shades. Fast and easy polishing reduces the time needed to achieve a natural finish and gloss.

\section{Optimum results when used together as a total Class II solution} These three Dentsply Sirona products are designed to work together for optimum adaptation to the dentist's technique and to the requirements of the restoration. The Palodent V3 matrix band provides the ideal natural contour and contact point for placing the restorative materials. The SDR material ensures that the natural contour of the matrix is incorporated into the final restoration with no gaps or voids in the proximal box. ceram.x universal fully adapts to the cavity and to the SDR layer while simplifying shade matching and finishing for a proper occlusal surface and efficient aesthetics.

Dentsply Sirona is proud to offer these three products as the integral components of a total Class II solution optimised for adaptability across every step of the procedure - an industry first.

1. van Dijken J W V. Class I and Class II SDR/CeramX mono+ restorations bonded with the one step self-etching adhesive XenoV+, five year report. Umeå, Sweden: Biomaterial Research Group, 15 February 2016. Released at the 2016 meeting of the International Association for Dental Research (IADR) in Seoul, Republic of Korea.

2. Substantiated by ceram.x universal user trial survey.

3. VITA is not a registered trademark of Dentsply Sirona, Inc
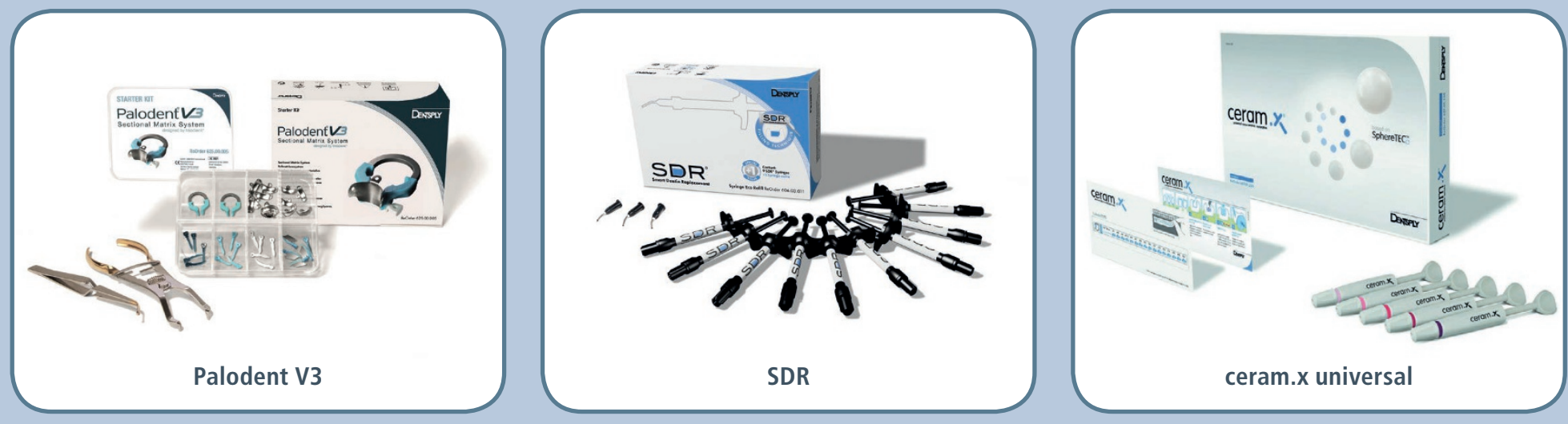